\title{
Rule-based extrapolation: A continuing challenge for exemplar models
}

\author{
STEPHEN E. Denton AND John K. KRUSChKe \\ Indiana University, Bloomington, Indiana \\ AND \\ Michael A. ERICKSON \\ University of California, Riverside, California
}

\begin{abstract}
Erickson and Kruschke $(1998,2002)$ demonstrated that in rule-plus-exception categorization, people generalize category knowledge by extrapolating in a rule-like fashion, even when they are presented with a novel stimulus that is most similar to a known exception. Although exemplar models have been found to be deficient in explaining rule-based extrapolation, Rodrigues and Murre (2007) offered a variation of an exemplar model that was better able to account for such performance. Here, we present the results of a new rule-plus-exception experiment that yields rule-like extrapolation similar to that of previous experiments, and yet the data are not accounted for by Rodrigues and Murre's augmented exemplar model. Further, a hybrid rule-and-exemplar model is shown to better describe the data. Thus, we maintain that rule-plus-exception categorization continues to be a challenge for exemplar-only models.
\end{abstract}

Single-system models of categorization posit a single representational system upon which all categorization is based. Some of the most successful single-system models suggest that categorical knowledge is exemplar based (Kruschke, 1992; Medin \& Schaffer, 1978; Nosofsky, 1986). Singlesystem models are certainly parsimonious and therefore deserving of continued study (Nosofsky \& Johansen, 2000). However, it has also been argued that aspects of human categorization abilities exceed the scope of exemplar-only models. The multiple-system models generally propose that humans have two types of category representation, one being instance based (or procedurally based) and the other being more abstract or rule based (Ashby, Alfonso-Reese, Turken, \& Waldron, 1998; Erickson \& Kruschke, 1998).

Rule-like behavior can be mimicked by exemplar models when categories are perfectly separable along a single feature dimension. But exemplar models have not fared well with some rule-plus-exception category structures. Erickson and Kruschke $(1998,2002)$ showed that people generalize category knowledge in a rule-like fashion when they are trained on a rule-plus-exception category structure. Importantly, novel stimuli that are similar to exception stimuli were generally classified in a rule-consistent manner. Because exemplar models use similarity to gauge category membership, they have difficulty making rulelike responses to stimuli that are near exceptions. Erickson and Kruschke's two studies used experimental evidence and model simulation to show that abstract, rule-like representations are needed, in addition to instance-based knowledge, to account for human performance on ruleplus-exception category structures. Since that time, Erickson (2008) has provided additional evidence via individual differences that people use multiple representations in category learning.

Recently, Rodrigues and Murre (2007) showed that an "extended" exemplar model can account for rule-like generalization. The model, called ALCOVEc, is an extension of ALCOVE (Kruschke, 1992) with two critical changes: the addition of an adaptive stimulus-specificity mechanism and a change in the response-mapping rule that causes the accentuation of small category-response differences. The addition of a specificity learning mechanism in ALCOVEc allows for stimulus-dependent similarity gradients. Exemplars that correspond to exception stimuli can become less similar to their neighboring stimuli and thus reduce exception generalization. Meanwhile, rule-compatible exemplars can become more similar to their neighbors and thus increase rule generalization. Rule-like behavior is generated from a number of exemplars that generalize broadly across psychological space. Rodrigues and Murre suggested that rule representations are not needed if exemplars are allowed to have adaptive specificities.

In this article, we present results from a new rule-plusexception experiment that poses difficult challenges for ALCOVEc. The new experiment was a simple variation of previous designs to which ALCOVEc was applied by Rodrigues and Murre (2007). Nevertheless, ALCOVEc fails to fit the results. The remainder of this article is organized as

S. E. Denton, sedenton@indiana.edu 
follows. First, we review some details of ALCOVEc. Next, we present the new rule-plus-exception experiment. Then, we will show that even the extended model cannot account for the data from our experiment. Further, we will show that ATRIUM, a hybrid rule-and-exemplar model (Erickson \& Kruschke, 1998), can better account for the data.

\section{ALCOVEc}

ALCOVE (Kruschke, 1992) and its extension ALCOVEc (Rodrigues \& Murre, 2007) assume that stimuli are represented as points in a multidimensional psychological space. An input node's activation, $a_{i}^{\text {in }}$, represents a value of the stimulus on the $i$ th dimension. An exemplar node in ALCOVE is activated on the basis of its similarity to the input stimulus. The similarity in turn depends on the distance between the exemplar and stimulus in psychological space, and the attention given to particular dimensions. The position of the $j$ th exemplar is denoted by the vector $\left\langle h_{j 1}, h_{j 2}, \ldots\right\rangle$, and the exemplar's activation is given by

$$
a_{j}^{\mathrm{ex}}=\exp \left(-c_{j} \sum_{i} \alpha_{i}\left|h_{j i}-a_{i}^{\mathrm{in}}\right|\right),
$$

where $\alpha_{i}$ is the attention strength of dimension $i$, and $c_{j}$ is the specificity of exemplar $j$. The specificity value for the $j$ th exemplar $\left(c_{j}\right)$ allows it to have a unique receptive field. Specificities for all exemplars are initialized at the same nonzero initial specificity value $\left(c_{\text {init }}>0\right)$. The larger the specificity, the narrower the receptive field of the exemplar.

Exemplar nodes are connected to output nodes with learnable association weights. Each output node represents one possible response category. Output node activation is given by

$$
a_{k}^{\text {out }}=\sum_{j} w_{k j} a_{j}^{\text {ex }}
$$

where $w_{k j}$ is the associative strength between exemplar $j$ and output category $k$.

In ALCOVEc (Rodrigues \& Murre, 2007), the output activations are mapped onto category response probabilities using the following power choice rule:

$$
p(K)=\frac{\left(a_{K}^{\text {out }}\right)^{\phi}}{\sum_{k}\left(a_{k}^{\text {out }}\right)^{\phi}},
$$

where $\phi$ is a response scaling constant or choice decisiveness parameter. This choice rule differs from ALCOVE, which instead uses

$$
p(K)=\frac{\exp \left(\phi a_{K}^{\text {out }}\right)}{\sum_{k} \exp \left(\phi a_{k}^{\text {out }}\right)},
$$

where $\phi$ is once again a response scaling constant. For both rules, larger $\phi$ values make the model output more decisive, whereby small activation differences are translated into larger choice preference differences. In general, the two choice rules produce similar results and differ only when there is sparse category evidence (i.e., all category activations are small). In this situation, the power rule can produce more decisive category responses. A related difference that will be relevant later when we discuss model fits arises when categories have zero or negative activation (i.e., $a_{K}^{\text {out }} \leq 0$ ). The power rule (Equation 3 ) cannot accept negative values, therefore negative output node activations are reset to zero (Rodrigues \& Murre, 2007). Zero activity in category node $K$ means that the probability of choosing category $K$ is zero according to the power rule (Equation 3). However, the exponential rule (Equation 4) assigns a nonzero choice probability to categories that have zero or negative activation.

All learning in ALCOVE and ALCOVEc is achieved through gradient descent on error, which is calculated with the use of a teacher value, $t_{k}$, which corresponds to the corrective feedback on a trial. In the present application, a strict teacher was used. The teacher value is 1 if the presented stimulus is a member of category $K$ and is zero otherwise. Association weights between exemplars and category nodes, as well as dimensional attentional strengths, are adjusted by moving down the error gradient. Association weights are initialized to zero, and attention strengths are initially all equal. These gradient descent equations, previously presented in Kruschke (1992), introduce two parameters, $\lambda_{w}$ and $\lambda_{\alpha}$, which are the output weight learning rate and the attention learning rate, respectively.

A second key extension made to ALCOVE to create ALCOVEc is the inclusion of specificity learning. Specificities are adjusted by gradient descent on error:

$$
\Delta c_{j}=-\lambda_{c}\left[\sum_{k}\left(t_{k}-a_{k}^{\text {out }}\right) w_{k j}\right] a_{j}^{\text {ex }}\left(\sum_{i} \alpha_{i}\left|h_{j i}-a_{i}^{\text {in }}\right|\right),
$$

where $\lambda_{c}$ is the specificity learning rate. Because negative specificities have no psychological interpretation, specificity values are reset to zero if Equation 5 drives them to negative values.

In fitting ALCOVE or ALCOVEc to human data, there is one other free parameter, dimensional salience, $v_{i}$. The salience of dimension $i$ multiplies the $h_{j i}$ and $a_{i}^{\text {in }}$ values by a constant scaling factor. The salience of one dimension can be set arbitrarily to 1 , so only $n-1$ saliences are freely estimated. The values of these parameters represent the relative salience of dimensions before any attention shifting.

\section{EXPERIMENT Learning a Rule With Exceptions}

We designed a simple rule-and-exception structure analogous to the one addressed previously by ALCOVEc (Rodrigues \& Murre, 2007). The training emphasized a few ruleobeying exemplars in the early trials, with the exceptional exemplars seamlessly introduced later in training. Participants were asked to classify simple stimuli into one of two categories. The stimuli were fixed-width rectangles whose height varied among eight equally spaced values. Within each rectangle, a vertical line segment was presented whose horizontal position varied among eight equally spaced val- 
Phase 1 (4 Blocks)

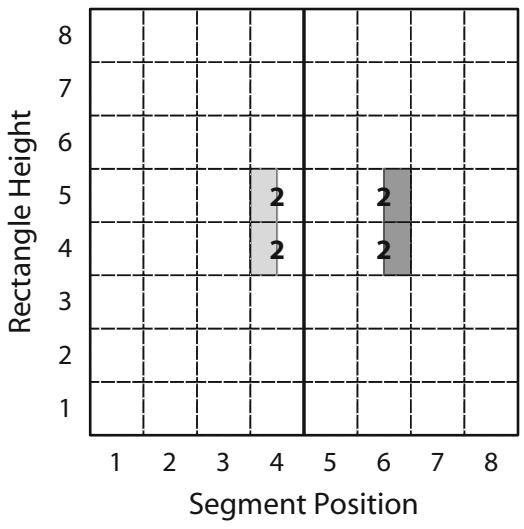

Phase 2 (2 Blocks)

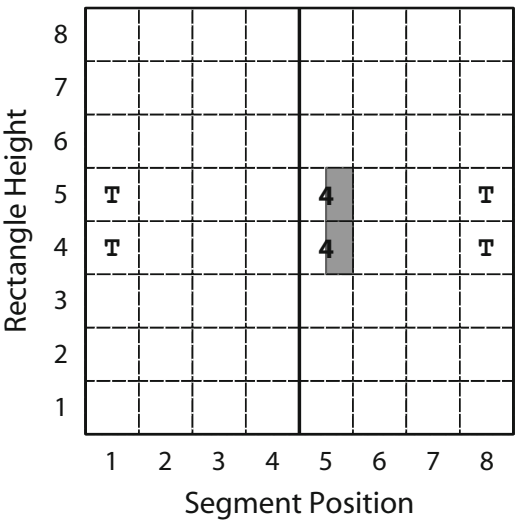

Phase 3 (2 Blocks)

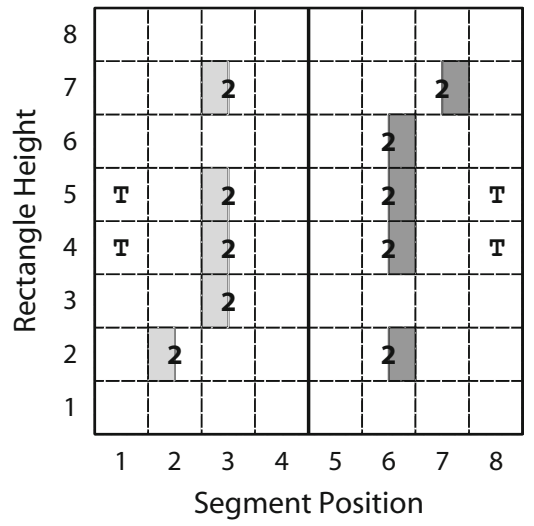

Phase 4 (4 Blocks)

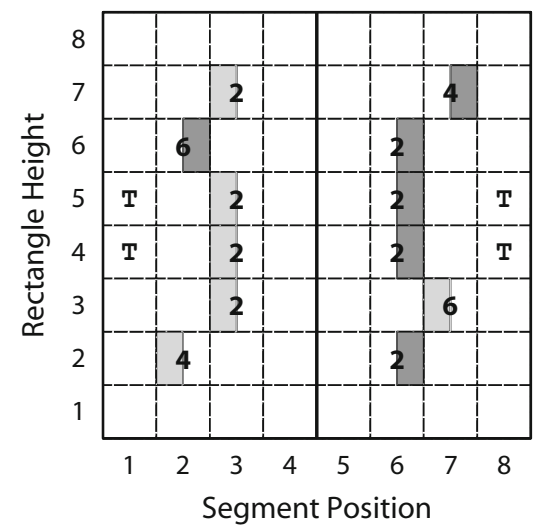

Phase 5 (2 Blocks)

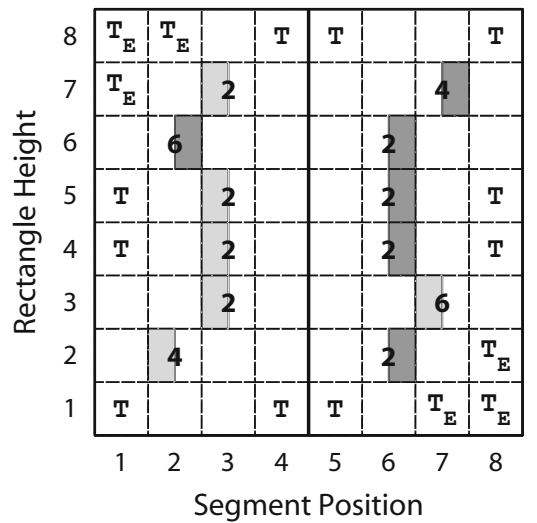

Figure 1. Category structure of the experiment. Each panel represents the $8 \times 8$ stimulus space for a phase of training. The rows in each panel represent stimulus values for the rectangle heights and the columns represent line segment positions. An empty cell indicates that the corresponding stimulus was not presented. Light gray indicates Category 1 stimuli, and dark gray indicates Category 2. A number within a cell indicates how many times the stimulus was presented per block. The number of blocks per phase is indicated at the top of each panel. Cells containing a $T$ indicate transfer stimuli that appeared once per block.

ues. The rectangle height and line segment position, when combined, produced a total of $64(8 \times 8)$ possible stimuli. The stimuli and the design are the same type used in experiments modeled by Rodrigues and Murre.

The layout of the two categories, across the five phases of the experiment, is presented in Figure 1. The categories can be distinguished generally by a simple one-dimensional rule that considers only the position of the line segment: Category 1 has left-side lines and Category 2 has right-side lines. The only two stimuli that do not conform to this rule appear in only the last phases of the experiment.

Training in Phase 1 begins with only four rule instances that cover only a limited region in the stimulus space. In Phase 2, training items are equally distant from (in between) those in Phase 1 but are members of a single category (Category 2). Phase 2 was intended to probe specificity learning by perturbing specificity symmetry of early learned exemplars. As a consequence, generalization from Category 2 exemplars should increase, while generalization from Category 1 exemplars should decrease. In Phase 3 , the rule is reinforced by presenting more stimuli that can be categorized correctly solely on the basis of line segment position. In Phase 4, the two stimuli that are exceptions to the rule (i.e., stimuli $\langle 2,6\rangle$ and $\langle 7,3\rangle$ in Figure 1) are introduced and intermixed with previously learned rule-consistent stimuli.

Phase 5 is similar to Phase 4 , but it includes a number of novel transfer stimuli that assay generalization from trained instances. These stimuli, labeled $T$ and $T_{E}$ in Figure 1, are important because they provide strong indicators of ruleversus-exception generalization. If participants use the rule to extrapolate to novel instances, then all of the transfer stimuli are classified as the same-side rule category. Participants generalizing by using exemplar knowledge tend to classify the transfer items nearest the exceptions $\left(T_{E}\right)$ as members of the opposite-side category. This assessment of extrapolation is the same sort used by Erickson and Kruschke (2002) and modeled by Rodrigues and Murre (2007).

\section{Method}

Participants. One-hundred forty students ( 71 females, 69 males) volunteered to participate for partial credit in an introductory psychology class at Indiana University. The average age of the participants was 19.8 years. 
Stimuli and Apparatus. The stimuli were displayed on a standard desktop computer in a dimly lit, ventilated, sound-dampened booth. They were presented in the center of the computer screen and a response prompt appeared on the left side of the screen. The height of the rectangle stimuli as they appeared on the screen varied between 3 and $18.5 \mathrm{~cm}$, separated in equal $2.2-\mathrm{cm}$ increments. The eight possible line segment positions varied by about $1.5 \mathrm{~cm}$. There were no tick marks on the margins (Erickson \& Kruschke, 2002). Participants made responses by pressing one of two keys ("F" and "J") on a standard keyboard.

Procedure. Written instructions for the categorization task were presented on the computer screen, and participants were allowed to read them at their own pace. The instructions explained that the task was to assign line drawings into one of two categories. Participants were told that they would occasionally see figures throughout the experiment for which there would be no corrective labels, and that they should make their best guess for such stimuli.

The details of the experimental design are presented in Figure 1. Each participant went through five phases that proceeded seamlessly through all trials. A total of 368 consecutive trials were presented, randomized within blocks. On each trial, a stimulus was presented and participants were prompted to press either "F" or "J." Participants were given unlimited time to respond. As soon as they responded, the correct category label was presented with an indication of whether their response for the current trial was accurate, except on a transfer trial, in which case the message "response recorded" was displayed. This feedback was displayed on the left side of the screen while the rectangle stimulus remained on the screen. If the response was correct, the participant was instructed to press the space bar to proceed to the next trial. If it was incorrect, the feedback was presented for $1 \mathrm{sec}$, followed by an instruction to press the space bar to proceed to the next trial.

\section{Results}

In this study, we were not interested in the guessing behavior of nonlearners and therefore we eliminated data from participants who, in the final training phase, could not correctly classify the training stimuli at better than chance levels. This criterion eliminated 29 participants, leaving 111 learners for further analysis. Overall, the average data from all of the participants were not extremely different from the average data from learners, and none of the trends in the data reversed when the excluded participants were retained.

Learners quickly attained high accuracy on the early rule-consistent stimuli. This suggests that learners adopted a rule, based on line segment position, to categorize the stimuli. This assertion is supported by Phase 3 responses, where learners achieved an average accuracy of $98.3 \%$ even though eight novel, rule-consistent stimuli appeared.

When the exception items appeared in Phase 4, overall accuracy was reduced and participants began to differ in how they responded. Because average data obscure differences among participants, a $k$-means clustering analysis was used to explore individual differences in participants' response profiles (see Erickson, 1999; Lewandowsky, Kalish, \& Griffiths, 2000; Webb \& Lee, 2004; Yang \& Lewandowsky, 2003). Profiles consisted of responses to all stimuli across all five phases of the experiment. By assigning each participant's response profile to the closest cluster using a Euclidean distance metric, the clustering analysis identified two distinct clusters of participants. A three-cluster solution was considered, but the third cluster contained only a few participants and was located between (and was a hybrid of) the other two, larger clusters. Therefore, we report only the two-cluster $k$-means solution in the remainder of this article.

The two clusters differed greatly in performance on the exception items. In Phase 5, the 45 participants in Cluster 1 responded correctly to exception items only $27.0 \%$ of the time, whereas the 66 participants in Cluster 2 responded correctly at a rate of $73.1 \%$. This difference of $46.1 \%$ is highly significant $[t(109)=12.82, p<.001, d=2.48$, $95 \% \mathrm{CI}=(39.0 \%, 53.2 \%)]$. Nevertheless, people in the two clusters performed about equally on the transfer stimuli that were close to the exceptions, labeled $T_{E}$ in Figure 1: $77.8 \%$ rule-consistent responses for Cluster 1 and $75.5 \%$ for Cluster 2. The proportion of rule-consistent responses on the $T_{E}$ items is significantly more than would be expected by chance alone $[t(110)=10.06, p<.001, d=0.96,95 \%$ $\mathrm{CI}=(71.2 \%, 81.6 \%)]$. The results for Cluster 2 are of particular importance because those participants learned both the rule and exceptions, and rule-plus-exception learning is the target behavior of the competing models.

\section{Model-Based Analysis}

The empirical results above suggest that participants generalized in a rule-based manner. These results echo those of Erickson and Kruschke (1998, 2002), and therefore, if Rodrigues and Murre's (2007) proposal is correct, these new data should be accommodated by ALCOVEc. In addition, a model with both rule and exemplar representations (ATRIUM; Erickson \& Kruschke, 1998) was fit to the data.

ATRIUM is a model composed of rule and exemplar modules. There is one rule module for each stimulus dimension that considers possible rules between all stimulus values. There is also an exemplar module that is identical to ALCOVE. The degree to which each module contributes to individual classification decisions is determined by a gating mechanism that learns which module performs the best and allows it to contribute the most. The details of ATRIUM's implementation can be found elsewhere (Erickson \& Kruschke, 1998) and therefore will not be discussed further here other than to note that in fitting the present experiment, a power choice rule was used instead of the original exponential choice rule. This change was made so that ATRIUM and ALCOVEc differ only in their representations and not in their choice rules.

Fitting considerations. The exact sequence of stimuli seen by each participant was presented to the models. The models were fit to the data, trial by trial, by minimizing the negative log likelihood. Various multipoint hill-climbing algorithms were used to ensure a thorough search of the parameter space for both models. For model comparison, complexity penalties were incorporated into the fitness measures (AIC and BIC), so that the higher parameter ATRIUM model would not have an unfair advantage compared with ALCOVEc.

When fitting the data trial by trial, we encountered a problem that was a direct result of the power response rule in ALCOVEc. Early in each simulated subject's training, when category activations are very slight, the model is likely to predict that the probability of choosing a par- 


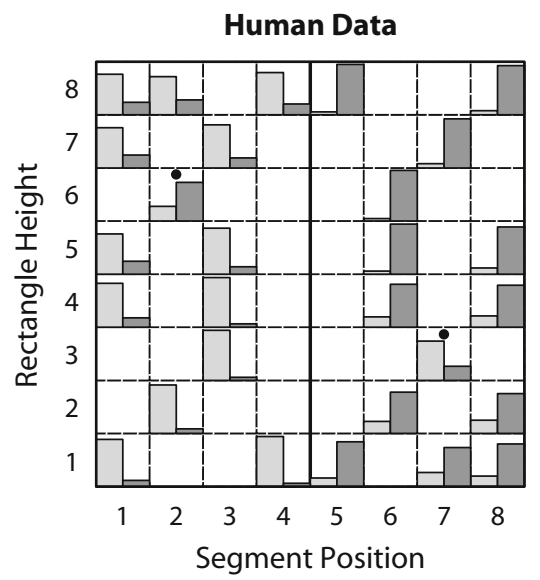

ATRIUM

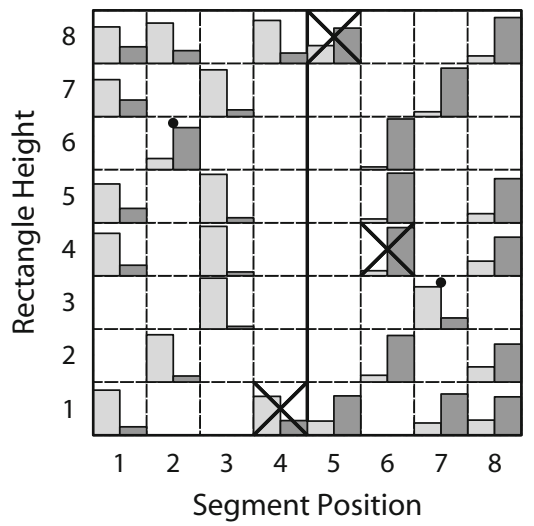

ALCOVEc

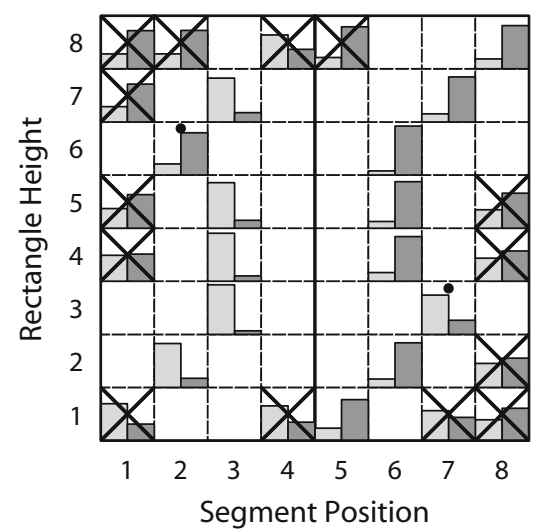

ALCOVEc (Fit Only to Phase 5)

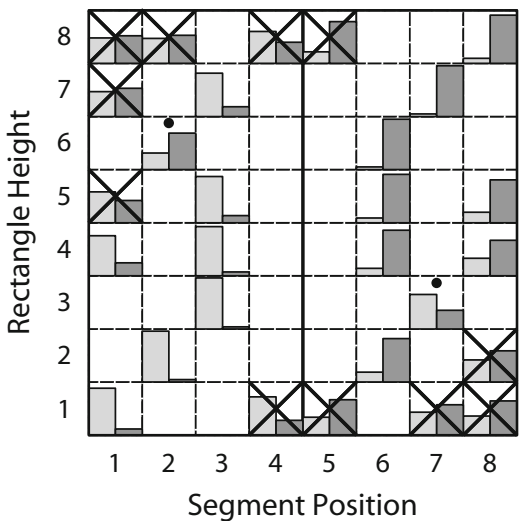

Figure 2. Results from Phase 5 for participants who learned the rule-plus-exceptions (Cluster 2) and the corresponding model predictions. As in Figure 1, the cells each represent a particular stimulus. The upper left panel shows human performance in Phase 5. Within each cell, the bars show the percentage of Category 1 (light gray) and Category 2 (dark gray) responses. Dots mark the locations of exception stimuli. The upper right and lower left panels show the Phase 5 predictions of ALCOVEc and ATRIUM, respectively, when the models are fit to all phases of the experiment. The lower right panel shows predictions of ALCOVEc when it is fit only to Phase 5. The Xs indicate stimuli for which the human response percentages are outside the $99 \%$ confidence interval for the model predictions.

ticular category is zero. If the human participant selects a category for which the model predicts zero probability, then the likelihood of the trial is zero, making the negative log likelihood infinite. To avoid this problem, the first block of Phase 1 (a total of eight trials per subject) was not included in the fit.

Due to the incremental nature of our experimental procedure, we assumed that exemplars in both models were recruited. The recruitment method was based solely on novelty; the first time a simulated subject encountered a particular stimulus, a corresponding exemplar would be stored by the simulated subject. Exemplars for both training stimuli and test stimuli were stored. Other recruitment schemes, including recruiting only training exemplars, storing training exemplars prior to learning, and storage of all stimuli, were also tried, and all produced qualitatively similar fits.

Fits of the models. Each model was fit to the two clusters of participant data separately. Because we are interested in how well the models account for rule-plusexception learning, we emphasize Cluster $2.1^{1}$ The Cluster 2 human data and corresponding model predictions for the testing phase (Phase 5) are presented in Figure 2. ATRIUM provides a better account of the data than does ALCOVEc. ${ }^{2}$ Inspection of the model predictions in Figure 2 indicates that ALCOVEc does not show robust rule extrapolation, unlike human participants (and ATRIUM). ALCOVEc makes several highly inaccurate predictions, marked by Xs in Figure 2. ALCOVEc's predictions for the $T_{E}$ transfer items are all at least $19 \%$ off and these predictions are often even ordinally wrong (i.e., showing a preference for the opposite-rule/exception category). ATRIUM shows only three cells marked by Xs, none of which is ordinally wrong and none of which involves extrapolation near exceptions.

Analysis of model behavior. Much of the reason that ALCOVEc has problems fitting these data is that humans can apparently adopt a rule for which they have seen only 
limited instances. People induce a rule early in training that they continue to apply throughout the experiment. ALCOVEc can emulate rule-like generalization when a number of the rule-consistent exemplars for both categories develop broad receptive fields (i.e., low specificities).

Our experiment challenges ALCOVEc and its specificity learning capabilities because early and late phases impose opposing constraints. For ALCOVEc to perform well on rule-plus-exception tasks, such as Phases 4 and 5 of our experiment, it must develop broad receptive fields on ruleconsistent exemplars and simultaneously develop narrow receptive fields on exception exemplars so that generalization is rule-like. Learning this specificity differentiation is made difficult for ALCOVEc by training it on a limited number of rule-consistent instances in Phases 1 and 2. Although humans have no trouble inferring a rule from a handful of instances, limiting early training to a few stimuli that are near the category boundary makes it unlikely that ALCOVEc will develop broad receptive fields that produce rule-like extrapolation similar to that of humans. When the full set of learning data is used, it is evident that ALCOVEc cannot simultaneously fit late training data and account for early learning.

Even when ALCOVEc is fit to Phase 5 alone, without being constrained by performance on earlier phases, it still does not predict robust rule-like extrapolation on novel transfer items, as can be seen in the lower-right panel of Figure $2 .{ }^{3}$ ALCOVEc shows only weak preferences on transfer items.

\section{SUMMARY AND DISCUSSION}

The empirical data and modeling results presented herein both point to a need for rule representation in categorization. The results from our rule-plus-exception experiment matched previous findings from Erickson and Kruschke $(1998,2002)$, that participants exhibited strong rule-like extrapolation, even to novel stimuli. These results could not be accounted for by the extended exemplar model, ALCOVEc, and were better explained by a hybrid rule-and-exemplar model, ATRIUM. Thus, the experimental data presented above provide a continuing challenge to theories that posit a single exemplar-based representational system.

The extended exemplar model, ALCOVEc, and specificity learning in particular, were proposed by Rodrigues and Murre (2007) as a means of producing the rule-consistent extrapolation seen in humans performing a rule-plus-exception task, but this mechanism is clearly insufficient. In the present experiment, ALCOVEc's predictions do not show robust rule extrapolation (see Figure 2). This is the case even though ALCOVEc uses a power-response rule that generally produces decisive category responses, even for novel stimuli.

As a historical note, specificity learning was included in the original ALCOVE code as an option (www.indiana .edu/ kruschke/publications.html\#ALCOVE). Erickson and Kruschke (1998) explored specificity learning in their modeling investigation and discovered that it did not enhance ALCOVE's fit to rule-plus-exception data. Specificity learning in ALCOVEc apparently worked for Rodrigues and Murre (2007) because of the inclusion of a different response rule. The combination of specificity learning and a power-choice rule was able to show some rule-like extrapolation, but we have shown that ultimately it still fails on a slightly different rule-plus-exception experiment.

Although it remains possible that a modified exemplar model, or an altogether different model with a single representational system, could account for our data, we continue to believe that multiple forms of representation are necessary. Erickson and Kruschke (1998, 2002) amassed a large amount of rule-plus-exception data. Ultimately, any candidate model must tackle all of these experiments, not just one phase of a single experiment.

\section{AUTHOR NOTE}

We thank Tammy Marnell, Chris Strait, and Lindsay Vogler for assistance in administering the experiment. Correspondence can be addressed to S. E. Denton, Department of Psychological and Brain Sciences, Indiana University, 1101 E. 10th St., Bloomington, IN 47405-7007 (e-mail: sedenton@indiana.edu).

\section{REFERENCES}

Ashby, F. G., Alfonso-Reese, L. A., Turken, A. U., \& Waldron, E. M. (1998). A neuropsychological theory of multiple systems in category learning. Psychological Review, 105, 442-481.

ERICKSON, M. A. (1999). Rule and exemplar representation in category learning (Doctoral dissertation, Indiana University, Bloomington, 1999). Dissertation Abstracts International, 60, 2377B.

Erickson, M. A. (2008). Executive attention and task switching in category learning: Evidence for stimulus-dependent representation. Memory \& Cognition, 36, 749-761.

Erickson, M. A., \& KruschKe, J. K. (1998). Rules and exemplars in category learning. Journal of Experimental Psychology: General, 127, 107-140.

Erickson, M. A., \& Kruschke, J. K. (2002). Rule-based extrapolation in perceptual categorization. Psychonomic Bulletin \& Review, 9, 160-168.

KrUschKe, J. K. (1992). ALCOVE: An exemplar-based connectionist model of category learning. Psychological Review, 99, 22-44.

Lewandowsky, S., Kalish, M., \& Griffiths, T. L. (2000). Competing strategies in categorization: Expediency and resistance to knowledge restructuring. Journal of Experimental Psychology: Learning, Memory, \& Cognition, 26, 1666-1684.

Medin, D. L., \& SchaffER, M. M. (1978). Context theory of classification learning. Psychological Review, 85, 207-238.

Nosofsky, R. M. (1986). Attention, similarity, and the identificationcategorization relationship. Journal of Experimental Psychology: General, 115, 39-57.

Nosofsky, R. M., \& Johansen, M. K. (2000). Exemplar-based accounts of "multiple-system" phenomena in perceptual categorization. Psychonomic Bulletin \& Review, 7, 375-402.

Rodrigues, P. M., \& Murre, J. M. J. (2007). Rules-plus-exception tasks: A problem for exemplar models? Psychonomic Bulletin \& Review, 14, 640-646.

Webb, M. R., \& LeE, M. D. (2004). Modeling individual differences in category learning. In K. Forbus, D. Gentner, \& T. Regier (Eds.), Proceedings of the 26th Annual Meeting of the Cognitive Science Society (pp. 1440-1445). Mahwah, NJ: Erlbaum.

YANG, L.-X., \& LEWANDOWSKY, S. (2003). Context-gated knowledge partitioning in categorization. Journal of Experimental Psychology: Learning, Memory, \& Cognition, 29, 663-679.

\section{NOTES}

1. The participants in Cluster 1 did not learn the exceptions and therefore did not exhibit learning behavior complex enough to warrant the extra mechanisms posited by ALCOVEc or ATRIUM. Consequently, the 
fits are of little interest, but are included for completeness. For Cluster 1, the fit value for ALCOVEc yielded AIC $=12,697$ and BIC $=12,744$ (for parameter values $c_{\text {init }}=0.3350, \phi=2.261, \lambda_{e}=0.03187, \lambda_{\alpha}=0.007668$, $\lambda_{c}=2.565 \times 10^{-11}$, and $\left.v_{1}=9.571\right)$. The fit value for ATRIUM yielded $\mathrm{AIC}=12,533$ and $\mathrm{BIC}=12,618$ (for parameter values $c=0.08878$, $\phi=2.638, \lambda_{e}=0.006304, \lambda_{\alpha}=0.03658, v_{1}=39.20, \gamma_{r}=34.33, \phi_{g}=$ $\left.5.047, \lambda_{r}=0.1062, \lambda_{g}=0.005578, \beta_{r 1}=-1.037, \beta_{r 2}=0.2328\right)$. For all fits, $v_{1}$ is the salience of dimension 1 (line segment position) and $v_{2}$ (corresponding to rectangle height) was set to 1 .

2. For Cluster 2, the fit value for ALCOVEc yielded AIC $=19,597$ and $\mathrm{BIC}=19,646$ (for parameter values $c_{\text {init }}=1.706, \phi=0.8532, \lambda_{e}=$ $0.008037, \lambda_{\alpha}=0.03608, \lambda_{c}=0.3491$, and $\left.v_{1}=2.754\right)$. The fit value for ATRIUM yielded AIC $=18,848$ and $\mathrm{BIC}=18,929$ (for parameter values $c=307.8, \phi=1.217, \lambda_{e}=0.4767, \lambda_{\alpha}=0$ [fixed], $v_{1}=0.9887$, $\gamma_{r}=2.799, \phi_{g}=15.94, \lambda_{r}=0.1085, \lambda_{g}=8.151 \times 10^{-4}, \beta_{r 1}=0.03937$, $\left.\beta_{r 2}=-0.06363\right)$. The BIC reduction of more than 700 is quite large for only four extra parameters, since BIC tends to favor models with fewer parameters. The main argument against ALCOVEc, however, is its qualitative failure revealed in Figure 2.

3. A thorough parameter search revealed a minimum negative log likelihood of 3,126.8 when ALCOVEc is fit only to Phase 5, Cluster 2 data. This best fit was obtained with parameter values: $c_{\text {init }}=1.087, \phi=$ $2.246, \lambda_{e}=0.01523, \lambda_{\alpha}=1.436 \times 10^{-10}, \lambda_{c}=0.1511$, and $v_{1}=1.758$. Because the number of data points included in this fit is far less than previous fits, AIC and BIC values are not comparable.

(Manuscript received September 28, 2007; revision accepted for publication February 21, 2008.) 